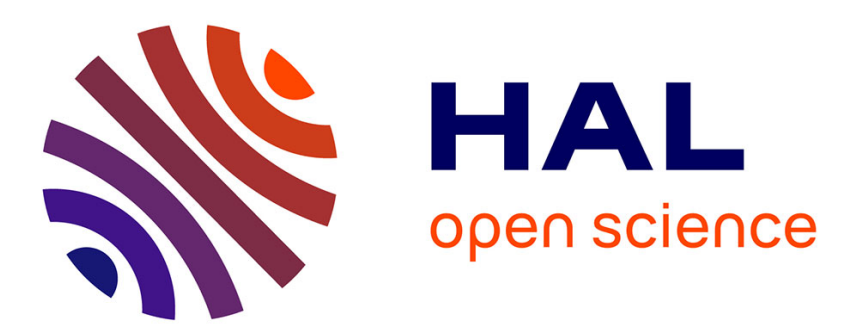

\title{
Appareil de mesure de capacité thermique et de transfert thermique jusqu'au mK
}

B. Hebral, G. Frossati, H. Godfrin, G. Schumacher, D. Thoulouze

\section{To cite this version:}

B. Hebral, G. Frossati, H. Godfrin, G. Schumacher, D. Thoulouze. Appareil de mesure de capacité thermique et de transfert thermique jusqu'au mK. Revue de Physique Appliquée, 1978, 13 (10), pp.533-545. 10.1051/rphysap:019780013010053300 . jpa-00244483

\section{HAL Id: jpa-00244483 https://hal.science/jpa-00244483}

Submitted on 1 Jan 1978

HAL is a multi-disciplinary open access archive for the deposit and dissemination of scientific research documents, whether they are published or not. The documents may come from teaching and research institutions in France or abroad, or from public or private research centers.
L'archive ouverte pluridisciplinaire HAL, est destinée au dépôt et à la diffusion de documents scientifiques de niveau recherche, publiés ou non, émanant des établissements d'enseignement et de recherche français ou étrangers, des laboratoires publics ou privés. 


\title{
APPAREIL DE MESURE DE CAPACITÉ THERMIQUE ET DE TRANSFERT THERMIQUE JUSQU'AU mK
}

\author{
B. HEBRAL, G. FROSSATI, H. GODFRIN, G. SCHUMACHER et D. THOULOUZE
}

Centre de Recherches sur les Très Basses Températures, C.N.R.S.

B.P. 166 X, 38042 Grenoble Cedex, France

(Reçu le 26 mai 1978, accepté le 12 juin 1978)

\begin{abstract}
Résumé. - Nous décrivons un appareillage composé d'une cellule de désaimantation adiabatique électronique attachée à un réfrigérateur à dilution, destiné à effectuer des mesures de capacité thermique et de transfert thermique entre 1,5 et $50 \mathrm{mK}$. Des mesures de capacité calorifique ont été effectuées sur les systèmes CMN-hélium. Une étude systématique en présence de ${ }^{3} \mathrm{He}$ liquide à pression nulle ou sous pression, ou d'un mélange $1,1 \%{ }^{3} \mathrm{He}-98,9 \%{ }^{4} \mathrm{He}$ permet de mesurer la capacité thermique du CMN jusqu'à environ 1,6 $\mathrm{mK}$. En présence de ${ }^{4} \mathrm{He}$ liquide, on met en évidence une anomalie de chaleur spécifique en-dessous de $10 \mathrm{mK}$ qui ne semble pas être interprétable par des considérations simples d'équilibre thermique. Nous déduisons également la capacité calorifique de ${ }^{3} \mathrm{He}$ superfluide des résultats obtenus avec ${ }^{3} \mathrm{He}$ liquide sous pression. Lors de mesures de transfert thermique à l'interface $\mathrm{CMN}$-mélange ${ }^{3} \mathrm{He}-{ }^{4} \mathrm{He}$, la variation en fonction de la température de la résistance thermique de contact est en assez bon accord avec d'autres résultats sur les poudres. Les résistances mesurées sont supérieures à celles prévues par le processus de phonons classique.
\end{abstract}

\begin{abstract}
We describe a cryogenic apparatus to perform heat capacity and heat transfer measurements in the range $1.5 \mathrm{mK}-50 \mathrm{mK}$. They are done in an adiabatic demagnetization cell attached to a dilution refrigerator. Heat capacity measurements were performed on $\mathrm{CMN}$-helium systems. Using liquid ${ }^{3} \mathrm{He}$ or a mixture $1.1 \%{ }^{3} \mathrm{He}-98.9 \%{ }^{4} \mathrm{He}$, we deduce the $\mathrm{CMN}$ specific heat above $1.6 \mathrm{mK}$. With liquid ${ }^{4} \mathrm{He}$, we observe a specific heat anomaly below $10 \mathrm{mK}$. It does not seem possible to interpret it by simple thermal equilibrium considerations. We also deduce the superfluid ${ }^{3} \mathrm{He}$ heat capacity from the results obtained with liquid ${ }^{3} \mathrm{He}$ under pressure. In the heat transfer measurements at the interface $\mathrm{CMN}$-mixture ${ }^{3} \mathrm{He}-{ }^{4} \mathrm{He}$, the temperature dependence of the thermal boundary resistance is in rather good agreement with other powder results. The measured resistances are larger than those predicted by the classical phonon process.
\end{abstract}

L'étude des propriétés physiques de l'hélium aux températures inférieures à $10 \mathrm{mK}$ a été entreprise il y a une douzaine d'années [1-2]. Elle mettait en œuvre une technique de double désaimantation adiabatique paramagnétique électronique, en partant d'une température d'environ $0,3 \mathrm{~K}$ obtenue à l'aide d'un réfrigérateur à ${ }^{3} \mathrm{He}$, et permettait d'atteindre des températures d'environ $3 \mathrm{mK}$.

Les améliorations dans la mise au point des réfrigérateurs à dilution de ${ }^{3} \mathrm{He}$ dans ${ }^{4} \mathrm{He}$ [3] ont conduit à l'utilisation des températures stables inférieures à $20 \mathrm{mK}$; un étage supplémentaire de désaimantation adiabatique paramagnétique électronique [4] ou nucléaire [5], ou de compression adiabatique de ${ }^{3} \mathrm{He}$ sur sa courbe de fusion [6] permettait alors d'obtenir une température d'environ $1-2 \mathrm{mK}$ et d'étudier les phases superfluides de ${ }^{3} \mathrm{He}$ par exemple.

Nous décrivons ici un appareil du type à désaimantation adiabatique paramagnétique électronique permettant des mesures de chaleur spécifique et de transfert thermique à l'interface solide-liquide jusqu'à $2 \mathrm{mK}$ environ. La source froide est un sel de nitrate de cérium et de magnésium (CMN) en poudre assurant le refroidissement de l'échantillon et de l'hélium liquide servant de contact thermique. Dans un premier temps, nous avons étudié le système $\mathrm{CMN}-\mathrm{He}$ seul. L'analyse des mesures de transfert thermique nécessite une bonne connaissance de la chaleur spécifique de chacun des deux milieux. L'ensemble des résultats précédemment obtenus sur le CMN présente une certaine dispersion à très basse température [7-11]; ne disposant pas des résultats nécessaires pour les phases superfluides de ${ }^{3} \mathrm{He}$ [4], nous avons effectué les mesures correspondantes qui sont présentées dans cet article, ainsi que les premiers résultats de transfert thermique à l'interface $\mathrm{CMN}$-mélange ${ }^{3} \mathrm{He}-{ }^{4} \mathrm{He}$.

Dans la première partie nous décrivons le montage 
cryogénique, la deuxième partie présentera ses performances et décrira la technique des mesures, la dernière partie étant consacrée aux résultats expérimentaux et à leur discussion.

1. Le montage cryogénique. - Il se compose d'un réfrigérateur à dilution d'un type original mis au point au laboratoire et d'une cellule de désaimantation adiabatique paramagnétique du $\mathrm{CMN}$, reliés par un interrupteur thermique supraconducteur. L'ensemble est représenté sur la figure 1.

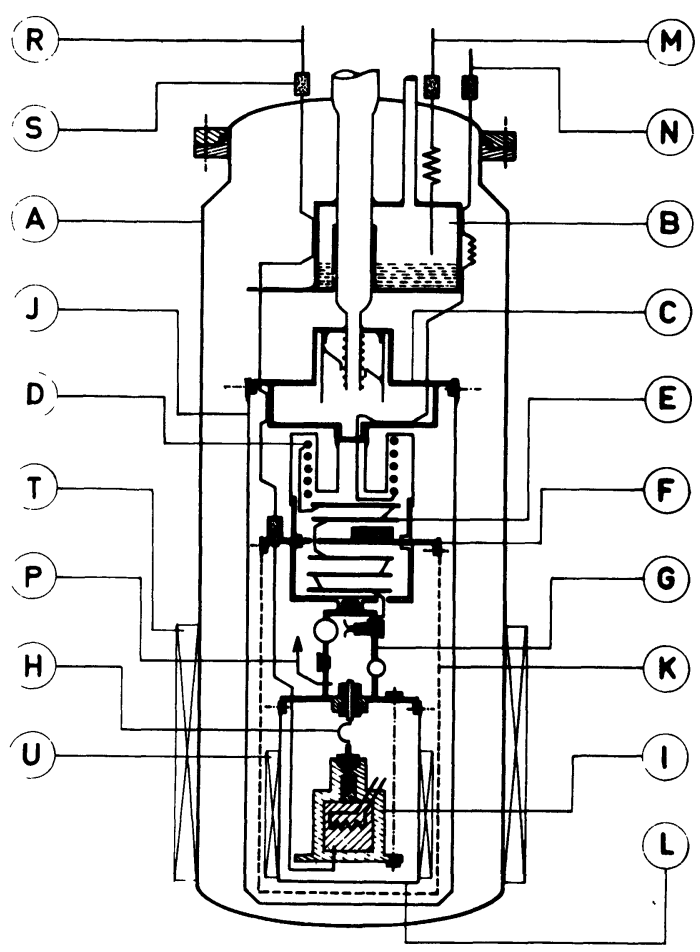

FIG. 1. - A) Calorimètre [Vacuum jacket]; B) Boîte $1 \mathrm{~K}[1 \mathrm{~K}$ box] ; C) Evaporateur [Still]; D) Echangeur $\mathrm{Cu}-\mathrm{Ni}[\mathrm{Cu}-\mathrm{Ni}$ exchanger] ; E) Echangeur plastique [Plastic exchanger] ; F) Plaque $30 \mathrm{mK}$ [30 mK plate]; G) Boîte à mélange [Mixing chamber]; H) Interrupteur étain [Tin switch]; I) Cellule à CMN [CMN cell];

$$
\left.\begin{array}{l}
\mathrm{J} \\
\mathrm{K}
\end{array}\right\} \text { Ecran [Shield] }\left\{\begin{array}{l}
0,5 \mathrm{~K} \\
30 \mathrm{mK} \\
15 \mathrm{mK} ;
\end{array}\right.
$$

M) Remplissage boîte $1 \mathrm{~K}$ [Filling tube $1 \mathrm{~K}$ box]; N) Phase concentrée [Concentrated phase]; P) Phase dilué [Dilute phase]; R) Remplissage cellule à $\mathrm{CMN}$ [Filling tube $\mathrm{CMN}$.cell]; $\mathrm{S}$ ) Cuivre ou argent fritté [Sintered copper or silver]; T) Bobine de champ principal [Main field coil]; U) Bobine de mutuelle inductance [Mutual inductance coil.]

L'appareil est supporté par un montage antivibratoire composé en particulier de trois bacs remplis de sable pesant environ 1 tonne chacun, posés sur des amortisseurs prévus pour couper l'effet des fréquences supérieures à $8 \mathrm{~Hz}$. Tous les tuyaux arrivant au cryostat comportent des liaisons souples. L'efficacité de ce dispositif semble suffisante : les vibrations autour du montage ne provoquent pas de réchauffement parasite, en particulier lors des mesures de chaleur spécifique. Le cryostat d'hélium liquide a une consommation de $0,41 / \mathrm{h}$ et une autonomie supérieure à $36 \mathrm{~h}$. Le niveau est déterminé au moyen d'une jauge à fil supraconducteur dont on mesure la résistance non immergée.

1.1 Le RÉFrigérateur a dilution. - 1.1.1 Pompage et condensation du mélange. - Le mélange composé de $101{ }^{3} \mathrm{He}$ et $401{ }^{4} \mathrm{He}$ TPN circule à l'aide d'une pompe à diffusion Bendix KS 200 et d'une pompe primaire Alcatel $2060 \mathrm{H}$ permettant d'assurer un débit maximum d'environ $200 \mu$ moles/s à une température de l'évaporateur d'environ $1 \mathrm{~K}$. Les caractéristiques de la ligne de pompage sont données dans l'annexe I. Le gaz, avant d'entrer dans le cryostat, passe par un piège à azote et à travers un débitmètre de masse à pont thermique, construit au laboratoire, qui permet de mesurer des débits variant de $10^{-6}$ à $10^{-3} \mathrm{~mole} / \mathrm{s}$.

Dans le vase hélium, le gaz ${ }^{3} \mathrm{He}$ passe sur des filtres de cuivre fritté. Il est ensuite condensé dans un tube de cuivre soudé sur la boîte à ${ }^{4} \mathrm{He}$ maintenue à $1,2 \mathrm{~K}$. Cette boîte est alimentée de façon continue à partir du bain à $4 \mathrm{~K}$ par l'intermédiaire d'un capillaire de $\mathrm{Cu}-\mathrm{Ni} \varnothing \quad 0,1-0,3 \mathrm{~mm}$, de $1,5 \mathrm{~m}$ de long $\left(Z \simeq 2 \times 10^{11} \mathrm{~cm}^{-3}\right)$. L'impédance assurant la détente de ${ }^{3} \mathrm{He}$ liquide se compose d'un capillaire, de $\mathrm{Cu}-\mathrm{Ni} \varnothing \quad 0,1-0,3 \mathrm{~mm}$, de $0,8 \mathrm{~m}$ de long $\left(Z \simeq 10^{11} \mathrm{~cm}^{-3}\right)$ placé entre la boîte à $1,2 \mathrm{~K}$ et l'évaporateur. Ce tube se trouve à l'intérieur d'un tube de $\mathrm{Cu}$ de $\varnothing 0,4-0,6 \mathrm{~mm}$ maintenu à la température de la boîte à $1,2 \mathrm{~K}$.

1.1.2 L'évaporateur. - Construit en cuivre, il offre un volume de $180 \mathrm{~cm}^{3}$ et est représenté sur la figure 2. Etudié il y a plusieurs années au labora-

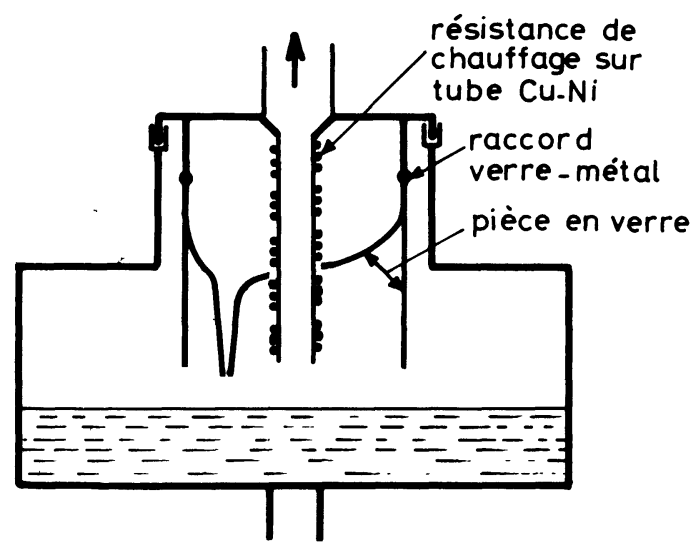

FIG. 2. - Evaporateur avec son dispositif pour brûler le film de ${ }^{4} \mathrm{He}$.

[Still with burning ${ }^{4} \mathrm{He}$ arrangement.]

toire [12], il comprend à l'intérieur un baffle en verre permettant de diminuer l'épaisseur du film superfluide et d'allonger sensiblement le chemin suivi par ce film. On sépare ainsi la région riche en ${ }^{4} \mathrm{He}$ de la région riche en ${ }^{3} \mathrm{He}$ où est effectué le pompage. Le film est ensuite évaporé sur le tube de pompage de $\mathrm{Cu}-\mathrm{Ni}(\varnothing 6 \mathrm{~mm})$ par une résistance de chauffage 
de $1100 \Omega$ en constantan $(\varnothing 5 / 100 \mathrm{~mm})$ qui sert également à chauffer l'évaporateur. Le gaz qui circule a ainsi une teneur affaiblie en ${ }^{4} \mathrm{He}$ comme on le voit sur la figure 3 , où l'on a représenté le pourcentage

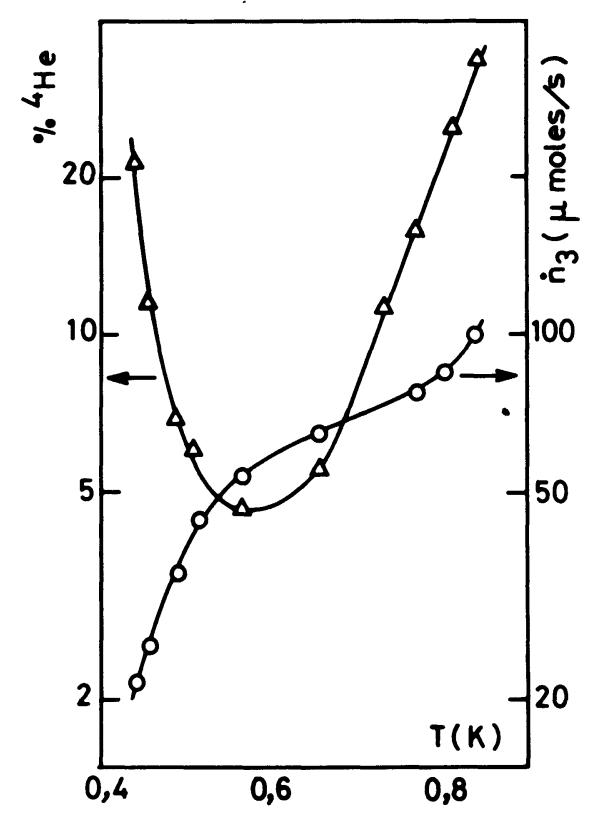

Fig. 3. - Pourcentage ${ }^{4} \mathrm{He}$ en sortie de l'évaporateur et débit ${ }^{3} \mathrm{He}$ dans le circuit de mélange en fonction de la température de l'évaporateur.

$\left[\%{ }^{4} \mathrm{He}\right.$ and ${ }^{3} \mathrm{He}$ flow rate as a fonction of the still temperature. $]$

de ${ }^{4} \mathrm{He}$ et le débit de ${ }^{3} \mathrm{He}$ en fonction de la température de l'évaporateur. L'évaporateur supporte également un écran en cuivre entourant toute la partie froide du cryostat.

1.1.3 Les échangeurs. - Le liquide ${ }^{3} \mathrm{He}$ entrant est thermalisé à la température de l'évaporateur dans un tube de cuivre soudé à ce dernier. Puis il est refroidi par la phase diluée dans un échangeur à contre-courant constitué de deux tubes concentriques en $\mathrm{Cu}-\mathrm{Ni}$ [13]. Dans le tube extérieur $(\varnothing 1-1,5 \mathrm{~mm})$ circule la phase diluée, le tube intérieur $(\varnothing 0,2-$ $0,4 \mathrm{~mm}$ ) étant traversé par la phase concentrée. La longueur de l'échangeur est de $1,5 \mathrm{~m}$.

Ce type d'échangeur tubulaire ne permet pas d'atteindre des températures inférieures à $20 \mathrm{mK}$, la surface d'échange étant trop faible. Pour obtenir des températures plus basses, d'autres types d'échangeurs offrant une surface d'échange bien supérieure sont nécessaires. Nous avons donc utilisé, à la suite de l'échangeur tubulaire, cinq échangeurs en Kapton d'un type précédemment mis au point au laboratoire [14].

Chaque échangeur est formé de canaux disposés en parallèle, comme le montre la figure 4 , dans lesquels circulent alternativement les phases concentrée et diluée. Chaque canal est séparé de son voisin par des feuilles de Kapton $50 \mathrm{H}$ de $12 \mu$ d'épaisseur [15]. La géométrie des canaux est obtenue par la super-

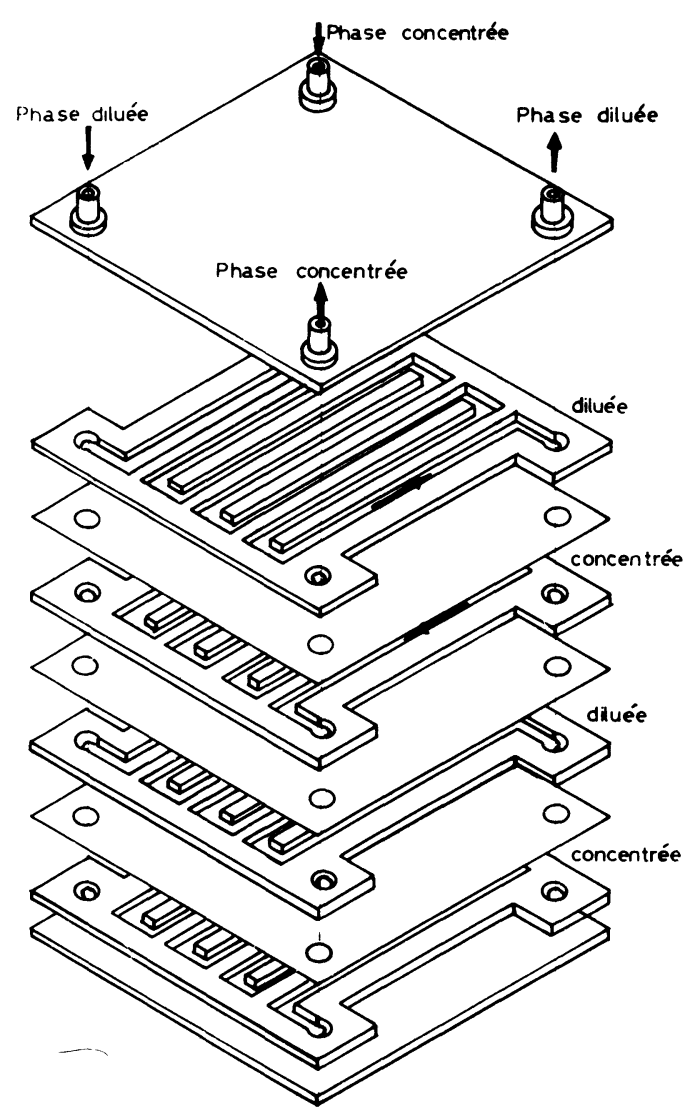

FIG. 4. - Montage des échangeurs plastiques. [Plastic exchangers arrangement.]

position d'espaceurs de Kapton préencollés GT 420 de $100 \mu$ d'épaisseur [16] découpés à la forme désirée. La surface d'échange pour chaque feuille est de $8 \mathrm{~cm}^{2}$. Le nombre d'espaceurs détermine l'impédance des canaux qui peut être ainsi ajustée pour limiter les effets de réchauffement visqueux et de conduction thermique dans le liquide. Espaceurs et feuilles collés ensemble à l'aide d'araldite DCY 230 (durcisseur HY 151) vont constituer un échangeur continu. Leurs caractéristiques géométriques sont données dans le tableau I; $\sigma$ est la surface d'échange totale, $Z_{d}$

\section{TABLEAU I}

Caractéristiques des échangeurs plastiques. $\sigma$ surface d'échange en $\mathrm{cm}^{2} ; Z_{\mathrm{d}}, Z_{\mathrm{c}}$ impédances diluées et concentrées en $\mathrm{cm}^{-3}$.

[Plastic exchangers characteristics. $\sigma$ exchange surface; $Z_{\mathrm{d}}, Z_{\mathrm{c}}$ diluted and concentrated impedances in $\mathrm{cm}^{-3}$.]

$$
\begin{array}{ll}
\sigma=16 \mathrm{~cm}^{2} & 2 \text { canaux dilués } \\
& 1 \text { canal concentré } \\
\sigma=32 \mathrm{~cm}^{2} & 3 \text { canaux dilués } \\
& 2 \text { canaux concentrés } \\
\sigma=32 \mathrm{~cm}^{2} & \begin{array}{l}
3 \text { canaux dilués } \\
2 \text { canaux concentrés }
\end{array} \\
\sigma=32 \mathrm{~cm}^{2} & \begin{array}{l}
3 \text { canaux dilués } \\
2 \text { canaux concentrés }
\end{array} \\
\sigma=32 \mathrm{~cm}^{2} & \begin{array}{l}
3 \text { canaux dilués } \\
2 \text { canaux concentrés }
\end{array}
\end{array}
$$


et $Z_{\mathrm{c}}$ les impédances côté dilué et côté concentré pour chaque échangeur. La surface d'échange totale est d'environ $150 \mathrm{~cm}^{2}$ dont $10 \mathrm{~cm}^{2}$ pour l'échangeur à contre-courant en $\mathrm{Cu}-\mathrm{Ni}$. Les échangeurs en Kapton mis en série sont divisés en deux groupes de deux et trois échangeurs séparés par une plaque de cuivre servant à la thermalisation des circuits des liquides et des fils de mesure. Cette plaque est refroidie par la phase diluée à l'aide d'une grande surface de cuivre fritté. Elle peut éventuellement supporter un écran en cuivre.

1.1.4 La boîte à mélange. - Réalisée dans un barreau d'araldite [17], elle a un volume d'environ $30 \mathrm{~cm}^{3}$. Sept bouchons coniques, en araldite, en facilitent l'accès : l'étanchéité est assurée par un bon rodage des parties mâle et femelle des cônes, recouvertes par un mince film de graisse aux silicones.

Un bouchon supporte un poral de cuivre fritté d'environ $900 \mathrm{~cm}^{2}$ sur lequel on peut placer quatre résistances à étalonner. Sur un autre bouchon on a fixé une résistance de $500 \Omega$ permettant de chauffer la boîte à mélange. Le troisième et le quatrième, ayant chacun un poral de cuivre fritté, sont destinés l'un à thermaliser un écran formé de fils de cuivre noyés dans de l'araldite entourant la cellule de désaimantation, l'autre à refroidir cette cellule via l'interrupteur thermique. Le cinquième bouchon supporte une petite bobine de mutuelle inductance dans laquelle on a placé $50 \mathrm{mg}$ de $\mathrm{CMN}$ utilisé pour la mesure de la température de la boîte à mélange. Ce sel est étalonné entre $1,3 \mathrm{~K}$ et $2 \mathrm{~K}$ en fonction de la tension de vapeur de ${ }^{4} \mathrm{He}$, après avoir admis du gaz d'échange dans le calorimètre. Un sixième bouchon reçoit le montage destiné à des mesures de résistivité électrique à l'aide d'un SQUID [18].

1.2 L'ÉTAGE DE DÉSAIMANTATION. - 1.2.1 L'interrupteur thermique et la bobine de champ. - La puissance frigorifique destinée à refroidir la cellule expérimentale est extraite de la boîte à mélange par l'intermédiaire d'un poral de cuivre fritté d'environ $4000 \mathrm{~cm}^{2}$ fixé sur une tige de cuivre de pureté $5 \mathrm{~N}$ de $3 \mathrm{~mm}$ de diamètre. Ce barreau traverse un bouchon conique, l'étanchéité étant assurée par du Stycast 2850 FT (catalyseur 24 LV).

Pour isoler la cellule contenant le CMN et l'He liquide de la boîte à mélange après désaimantation et pour assurer le contact thermique pendant le prérefroidissement de la cellule, on utilise un interrupteur thermique supraconducteur.

Il est constitué d'une feuille d'étain de pureté $5 \mathrm{~N}$ de $0,3 \times 5 \times 20 \mathrm{~mm}$ soudée au métal de Wood d'une part sur la tige de cuivre venant de la boîte à mélange, d'autre part sur une tige de cuivre analogue pénétrant par un bouchon conique dans la cellule de désaimantation adiabatique. Cette seconde tige est soudée à un poral d'argent fritté offrant une surface d'échange estimée à $600 \mathrm{~cm}^{2}$. L'interrupteur thermique, commandé par le champ de la bobine de désaimantation, n'est ouvert qu'une heure environ après le début de celle-ci. Néanmoins l'apport de chaleur sur la cellule est suffisamment faible pendant ce temps pour ne pas limiter la température finale, compte tenu de la résistance de Kapitza aux températures inférieures à $20 \mathrm{mK}$.

La bobine de champ de diamètre $141 \mathrm{~mm}$ et de longueur $310 \mathrm{~mm}$ est placée sur le calorimètre dans le bain de ${ }^{4} \mathrm{He}$ à 4,2 K. 2250 spires de fil de $\mathrm{Nb}-\mathrm{Ti}$ multifilamentaire cuivré sont bobinées en deux couches et permettent d'obtenir un champ d'environ $2300 \mathrm{G}$ avec un courant de $25 \mathrm{~A}$. La bobine peut être fermée sur elle-même grâce à un court-circuit supraconducteur commandé par une résistance de chauffage d'environ $100 \Omega$ parcourue par un courant de $40 \mathrm{~mA}$.

1.2.2 La cellule de désaimantation. - Réalisée également en araldite, elle a une paroi de $8 \mathrm{~mm}$ destinée à supporter des pressions d'environ 50 bars. Elle est supportée par la boîte à mélange au moyen de trois tiges de nylon d'environ $10 \mathrm{~cm}$ de longueur et $3 \mathrm{~mm}$ de diamètre. Elle contient $28,5 \mathrm{~g}$ de CMN broyé en grains de dimension inférieure à $50 \mu$ $(\varnothing$ moyen $30 \mu)$ et tassé à $81 \%$ sous la forme d'un cylindre de diamètre $28 \mathrm{~mm}$ et de hauteur $28 \mathrm{~mm}$.

Le capillaire de remplissage en $\mathrm{Cu}-\mathrm{Ni}$ est formé de $26 \mathrm{~cm}$ de $\varnothing 0,1-0,3 \mathrm{~mm}$ entre la boîte à $1 \mathrm{~K}$ et l'évaporateur, de $1 \mathrm{~m}$ de $\varnothing 0,2-0,6 \mathrm{~mm}$ entre l'évaporateur et la plaque intermédiaire et de $60 \mathrm{~cm}$ de $\varnothing \quad 0,2-0,6 \mathrm{~mm}$ entre cette plaque et la cellule. Un filtre en cuivre fritté thermalise le liquide à $4,2 \mathrm{~K}$ et permet d'éviter de boucher le capillaire. Des filtres d'argent fritté placés sur l'évaporateur et la plaque froide assurent la thermalisation de ce liquide. Ces thermalisations sont indispensables en particulier pour faire des mesures avec ${ }^{4} \mathrm{He}$ liquide et minimiser ainsi les apports de chaleur par conduction dans le liquide.

Pour les mesures sous pression avec ${ }^{3} \mathrm{He}$ liquide ou solide, on utilise le système de mise sous pression classique avec cryopompe à $4,2 \mathrm{~K}$ décrit dans la référence [19].

Une résistance de carbone Speer $100 \Omega$ coupée à une épaisseur de $300 \mu$ est placée au fond de la cellule et permet d'évaluer la température pendant le prérefroidissement sous champ magnétique.

Après désaimantation, la mesure de la susceptibilité du sel de CMN fournit la température de la cellule. C'est cette température magnétique $T^{*}$ déduite de la susceptibilité du CMN par une loi de Curie que nous utilisons dans cet article.

Une résistance de chauffage de $60 \Omega$ de fil d'Evanohm de diamètre $50 \mu$ est noyée dans le $C M N$ pendant le compactage. Elle permet de chauffer l'ensemble $\mathrm{CMN}-\mathrm{He}$ lors des mesures de chaleur spécifique ou de transfert thermique.

2. Résultats cryogéniques et techniques de mesure. - Après avoir présenté les performances de l'appareil à dilution, nous décrivons la façon dont sont conduites 
les désaimantations et les mesures de chaleur spécifique.

2.1 Performances De L'APPAReIL A DILUTION. Ses performances sont principalement conditionnées par la surface d'échange. Pour un échangeur continu parfait, on montre [20] que la température $T_{\mathrm{m}}$ de la boîte à mélange est liée au débit $\dot{n}$ par :

$$
T_{\mathrm{m}}^{2}=12,8 \times 10^{-6} R_{\mathrm{K}} \frac{\dot{n}}{S}
$$

où $S$ est la surface d'échange et $R_{\mathrm{K}}$ le coefficient de résistance de Kapitza entre la phase diluée et la paroi. Plus généralement, si l'on tient compte des pertes $\dot{Q}$ sur la boîte à mélange, $T_{\mathrm{m}}$ est donné par :

$$
T_{\mathrm{m}}^{2}=12,8 \times 10^{-6} R_{\mathrm{K}} \frac{\dot{n}}{S}+1,2 \times 10^{4} \frac{\dot{Q}}{\dot{n}}
$$

$T$ en $\mathrm{K}, R_{\mathrm{K}}$ en $\mathrm{cm}^{2} . \mathrm{K}^{4} / \mathrm{W}, \dot{n}$ en $\mu$ mole $/ \mathrm{s}, \dot{Q}$ en $\mathrm{W}, S$ en $\mathrm{cm}^{2}$.

Sur la figure 5 , on a représenté la température $T_{\mathrm{m}}$ de la boîte à mélange en fonction du rapport de la surface d'échange $S$ au débit $\dot{n}_{3}$ de ${ }^{3} \mathrm{He}$. La tempé-

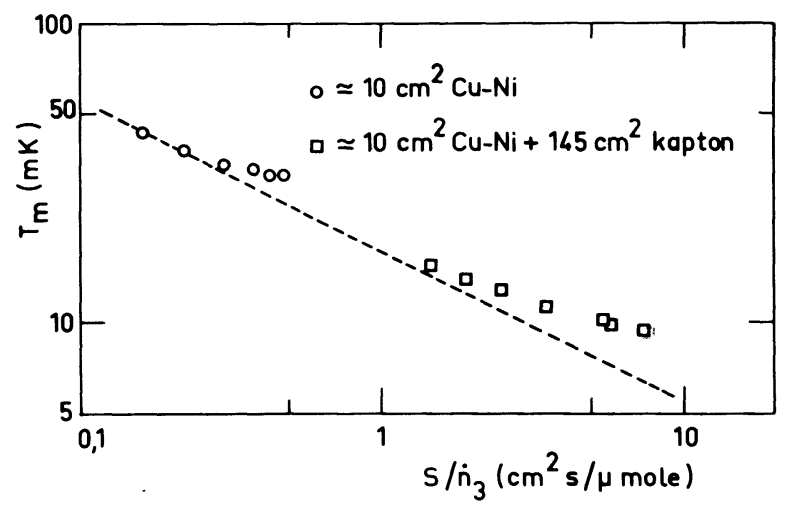

Fig. 5. - Température de la boîte à mélange en fonction de $S / \dot{n}_{3}$. La droite correspond à l'échangeur parfait défini dans le texte.

[Mixing chamber temperature versus $S / \dot{n}_{3}$. The dashed line corresponds to the ideal heat exchanger defined in the text.]

rature minimale atteinte avec l'échangeur tubulaire en $\mathrm{Cu}-\mathrm{Ni}$ est de $25 \mathrm{mK}$ environ; en ajoutant les échangeurs plastiques, elle devient inférieure à $10 \mathrm{mK}$.

Sur la figure 5 , on a aussi représenté la loi (1) de l'échangeur parfait qui est bien suivie au-dessus de $35 \mathrm{mK}$ avec l'échangeur $\mathrm{Cu}-\mathrm{Ni}$ seul et au-dessus de $15 \mathrm{mK}$ lorsqu'on ajoute les échangeurs plastiques ; en dessous de $35 \mathrm{mK}$ et $15 \mathrm{mK}$ respectivement, l'écart à la loi (1) de l'échangeur parfait lors de nos mesures à faible débit montre l'influence des pertes sur la boîte à mélange qui, calculées à partir de (2), sont d'environ $1 \mathrm{erg} / \mathrm{s}$ à $20 \mu$ moles/s et $9,5 \mathrm{mK}$. Cet écart est aussi montré sur la figure 6 où l'on a représenté la charge thermique appliquée sur la boîte à mélange en fonction de la température de la boîte pour trois

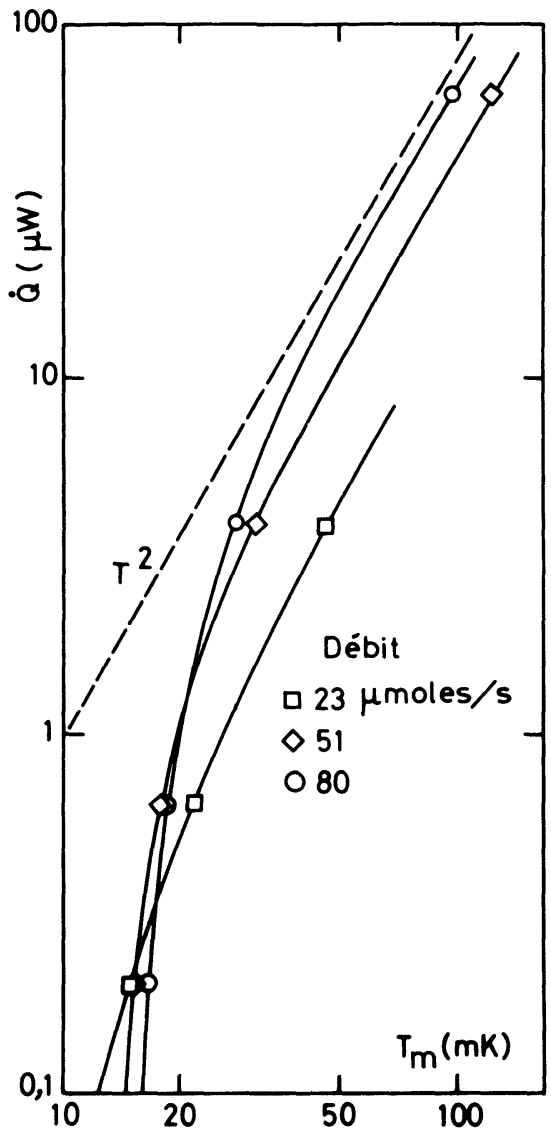

Fig. 6. - Charge thermique appliquée à la boîte à mélange en fonction de $T_{\mathrm{m}}$ pour trois débits différents.

[Applied power on the mixing chamber as a function of $T_{\mathrm{m}}$ for three different flow rates.]

débits différents. Les pertes déduites de ces courbes sont en bon accord avec celles déterminées à partir des résultats montrés sur la figure 5 : environ $3 \mathrm{erg} / \mathrm{s}$ à $23 \mu \mathrm{moles} / \mathrm{s}$ et $12 \mathrm{mK}$.

Il se trouve que pour ces échangeurs, le coefficient $R_{\mathrm{K}}$ de résistance de Kapitza est le même pour l'échangeur $\mathrm{Cu}-\mathrm{Ni}$ seul que pour les échangeurs plastiques : $R_{\mathrm{K}}=25 \mathrm{~cm}^{2} \cdot \mathrm{K}^{4} / \mathrm{W}$. Cette valeur pour l'échangeur $\mathrm{Cu}-\mathrm{Ni}$ est en bon accord avec les valeurs obtenues récemment pour des alliages $\mathrm{Cu}-\mathrm{Be}$ ou $\mathrm{Cu}-\mathrm{Cr}$ : $15<R_{\mathrm{K}}<30 \mathrm{~cm}^{2} . \mathrm{K}^{4} / \mathrm{W}$ [21]. Pour les échangeurs plastiques, cette valeur est aussi en bon accord avec celle précédemment obtenue pour d'autres échangeurs du même type : $R_{\mathrm{K}}=18 \mathrm{~cm}^{2} . \mathrm{K}^{4} / \mathrm{W}$ [14] ou avec celle déduite de mesures sur de l'Epibond $100 \mathrm{~A}$ : $R_{\mathrm{K}}=30 \mathrm{~cm}^{2} \cdot \mathrm{K}^{4} / \mathrm{W}$ [22]. Ces résultats indiquent également que, dans nos échangeurs, tous les canaux contribuent à l'échange, c'est-à-dire que les canaux en parallèle offrent des impédances comparables; dans le cas contraire, on surestimerait $S$ et on obtiendrait une valeur anormalement élevée de $R_{\mathrm{K}}$.

2.2 Procédure EXPÉRIMENTALE DE DÉSAIMANTATION ET RÉSULTATS CRYOGÉNIQUES. - La cellule contenant le CMN remplie avec l'hélium liquide ou solide à environ $1,3 \mathrm{~K}$ est alors mise sous champ 
magnétique. Avec ${ }^{3} \mathrm{He}$ liquide, ${ }^{3} \mathrm{He}$ solide ou un mélange ${ }^{3} \mathrm{He}-{ }^{4} \mathrm{He}$ à $1 \%$ de ${ }^{3} \mathrm{He}$, les essais ont montré que le champ initial $H_{0}$ optimal pour effectuer la désaimantation est de $1 \mathrm{kG}$. La température initiale de $15 \mathrm{mK}$ est atteinte en $36 \mathrm{~h}$ avec le mélange, alors qu'il faut 4 jours avec ${ }^{3} \mathrm{He}$ sous 29 bars : ceci est lié à la grande chaleur spécifique et à la mauvaise conductivité thermique de ${ }^{3} \mathrm{He}$ sous pression $[2,23]$. Avec ${ }^{4} \mathrm{He}$, la température initiale était de $20 \mathrm{mK}$ par suite de la grande résistance de Kapitza entre l'argent fritté et le liquide et de la très faible conductivité thermique de ${ }^{4} \mathrm{He}$ dans les pores du CMN à cette température; ceci a conduit à utiliser un champ initial $H_{0}$ d'environ $2 \mathrm{kG}$ dans ce cas.

La désaimantation se fait en trois étapes :

$$
H_{0} \rightarrow \frac{H_{0}}{2}, \frac{H_{0}}{2} \rightarrow \frac{H_{0}}{4}, \quad \frac{H_{0}}{4} \rightarrow 0
$$

de $1 \mathrm{~h}, 20 \mathrm{~min}$. et $40 \mathrm{~min}$. respectivement. Avec ${ }^{3} \mathrm{He}$ liquide, ${ }^{4} \mathrm{He}$ liquide ou le mélange, l'équilibre thermique est atteint en quelques minutes après la fin de la désaimantation. Avec ${ }^{3} \mathrm{He}$ solide, il n'est obtenu qu'après environ $2 \mathrm{~h}$.

Les pertes sur la cellule sont d'environ 1,5 erg/min. après la première désaimantation. Cette valeur tend à diminuer après plusieurs cycles aimantation-désaimantation pour atteindre $0,5 \mathrm{erg} / \mathrm{min}$. après environ quatre semaines d'expérience. Dans le cas des mesures avec ${ }^{4} \mathrm{He}$ liquide, les pertes sont plus élevées : environ $2 \mathrm{ergs} / \mathrm{min}$.

Le taux de compactage des grains de CMN était choisi pour que l'on puisse atteindre sa température d'ordre et donc étudier les phases superfluides de ${ }^{3} \mathrm{He}$ liquide. Il est nécessaire pour cela que la variation d'entropie du CMN sous champ pendant le prérefroidissement de la cellule soit nettement supérieure à la variation d'entropie de ${ }^{3} \mathrm{He}$ pendant la désaimantation. Il faut aussi éviter un taux de compactage trop élevé, conduisant à utiliser très peu de ${ }^{3} \mathrm{He}$, ce qui rendrait trop difficile l'analyse des résultats de capacité calorifique, celle de ${ }^{3} \mathrm{He}$ étant complètement masquée par celle du CMN. Ceci conduit à utiliser une cellule dont le taux de compactage est compris entre 75 et $85 \%$ pour un champ initial de $1000 \mathrm{G}$ et une température initiale d'environ $15 \mathrm{mK}\left({ }^{1}\right)$. Dans la cellule décrite ici pour un taux de compactage de $81 \%$ et dans ces conditions initiales, l'entropie de ${ }^{3} \mathrm{He}$ sous 29,2 bars était de $8 \times 10^{5} \mathrm{ergs} / \mathrm{K}$ et la variation d'entropie du CMN de $2 \times 10^{6} \mathrm{ergs} / \mathrm{K}$.

$\left({ }^{1}\right)$ Cette valeur dépend aussi de la géométrie de la cellule : l'une des cellules que nous avons utilisée, analogue à celle décrite ici, était munie d'une tour de $2 \mathrm{~cm}$ ne contenant que ${ }^{3} \mathrm{He}$; le taux de compactage du sel de désaimantation était de $75 \%$. Cette cellule n'atteignait que $T^{*}=2,12 \mathrm{mK}$ à 29 bars et $1,85 \mathrm{mK}$ à 0 bar à cause de la mauvaise diffusivité thermique de ${ }^{3} \mathrm{He}$ au début de la désaimantation. De même une troisième cellule, au taux de compactage de $60 \%$, sans tour contenant du liquide, descend à $T^{*}=1,85 \mathrm{mK}$ avec ${ }^{3} \mathrm{He}$ sous pression nulle.
Avec ${ }^{3} \mathrm{He}$ liquide sous 20 bars ou 0 bar et le mélange ${ }^{3} \mathrm{He}-{ }^{4} \mathrm{He}$, on atteint la température d'ordre du CMN caractérisée par un léger maximum de la susceptibilité : $T^{*}=1,45 \mathrm{mK}$. Avec ${ }^{4} \mathrm{He}$ liquide ou ${ }^{3} \mathrm{He}$ solide, la température minimale atteinte est d'environ $1,7 \mathrm{mK}$. La chaleur spécifique de ${ }^{3} \mathrm{He}$ solide varie en effet en $T^{-2}$ dans cette gamme de température et celle d'un liquide de Fermi en $T$, ce qui donne une charge thermique plus élevée dans le cas du solide. Pour ${ }^{4} \mathrm{He}$ liquide; la température finale de $1,7 \mathrm{mK}$ s'explique probablement par une température initiale plus élevée. Les faibles pertes arrivant sur la cellule permettent de rester environ $20 \mathrm{~h}$, à 29 bars, en dessous de la température de transition superfluide, dans le cas où le réchauffement est dû uniquement aux pertes.

\subsection{TECHNIQUE DE MESURE DE CHALEUR SPÉCIFIQUE.} - On utilise une technique classique de mesure quasi-adiabatique, la cellule étant isolée de la boîte à mélange par l'interrupteur supraconducteur. Pendant un intervalle de temps $\Delta t$, on fait passer un courant $I$ dans le fil de chauffage placé dans la cellule. La chaleur spécifique est directement donnée par le rapport de la quantité de chaleur $\Delta Q$ à l'élévation $\Delta T$ de température $C=\Delta Q / \Delta T$.

2.3.1 Mesure de la quantité de chaleur. - La résistance de chauffage est mesurée à $4,2 \mathrm{~K}$ au début et à la fin du cycle de mesures ainsi qu'à environ $30 \mathrm{mK}$ à l'aide d'un pont de Wheatstone travaillant en courant alternatif. On vérifie ainsi que sa valeur reste constante : 60,0 $\Omega$. Le courant de chauffage est fourni par une alimentation pouvant délivrer des créneaux d'intensité $I$ variant entre $0,1 \mu \mathrm{A}$ et $8 \mathrm{~mA}$ pendant un intervalle de temps $\Delta t$ compris entre $1 \mathrm{~ms}$ et $10000 \mathrm{~s}$. La valeur de $\Delta Q$ est choisie pour que l'élévation de température correspondante $\Delta T$ soit telle que $\Delta T / T \leqslant 10 \%$. La plupart des points pour $T<10 \mathrm{mK}$ sont tels que : $\Delta T / T<1 \%$. Les énergies $\Delta Q$ sont comprises entre 3 et 1000 ergs dissipés pendant une durée $\Delta t$ variant entre $0,01 \mathrm{~s}$ et $100 \mathrm{~s}$.

2.3.2 Mesure de la température. - Elle est déduite de la susceptibilité du CMN mesurée à l'aide d'un pont de mutuelle inductance, travaillant à $119 \mathrm{~Hz}$, mis au point au laboratoire [24]. Le courant primaire de $0,1 \mathrm{~mA}$ crée un champ d'environ $15 \mathrm{mOe}$. La variation maximale de mutuelle inductance est d'environ $600 \mu \mathrm{H}$. Le primaire de la mutuelle est formé d'une couche de 1250 tours de fil Nb-Ti $(\varnothing 0,05 \mathrm{~mm})$ cuivré monofilamentaire de longueur $100 \mathrm{~mm}$, bobinés sur l'écran de diamètre $83 \mathrm{~mm}$ entourant la cellule de désaimantation et lié à la boîte à mélange. Le secondaire en cuivre $(\varnothing 0,05 \mathrm{~mm})$ est bobiné sur le primaire en trois bobinages jointifs de longueur totale $50 \mathrm{~mm}$ de façon à minimiser la mutuelle à vide : les deux bobines extrêmes de $10 \mathrm{~mm}$ de long et 380 tours en 3 couches chacune sont bobinées en sens contraire de l'enroulement central de $30 \mathrm{~mm}$ de long et 760 tours en 2 couches. Les centres des 
bobinages primaire et secondaire et le centre du cylindre de CMN sont confondus.

L'étalonnage de ce $\mathrm{CMN}$ ne peut être effectué entre 1 et $2 \mathrm{~K}$ à l'inverse de celui placé dans la boîte à mélange. En effet, dans cette gamme de température, à la susceptibilité du CMN de désaimantation se superpose celle de l'environnement : tubes de remplissage, soudures devenant supraconductrices, etc. Tout ceci est mesuré par la bobine de mutuelle inductance qui a un diamètre trois fois plus grand que celui $\mathrm{du} C \mathrm{CMN}$. C'est pourquoi l'étalonnage est fait entre 10 et $150 \mathrm{mK}$ après désaimantation en fonction de la résistance de carbone d'étalonnage connu, placée dans la cellule. Cette méthode, bien qu'indirecte, donne de bons résultats en général. L'incertitude due à l'étalonnage sur la mesure de température est inférieure à $1 \%$. Les constantes d'étalonnage sont reproductibles d'une expérience à l'autre quel que soit l'environnement : ${ }^{3} \mathrm{He}$ liquide sous pression ou sous pression nulle, mélange à $1,1 \%{ }^{3} \mathrm{He},{ }^{3} \mathrm{He}$ solide. Une confirmation de la validité de notre étalonnage est donnée par la détermination de la température de transition superfluide de ${ }^{3} \mathrm{He}$ : l'accord avec les expériences antérieures [4] est bon comme on le verra plus loin. Néanmoins nous avons observé une variation notable de l'étalonnage $(\sim 10 \%)$ lors des expériences avec ${ }^{4} \mathrm{He}$ liquide; en outre dans ce dernier cas, comme pour les mesures avec ${ }^{3} \mathrm{He}$ solide, l'étalonnage n'est effectué qu'audessus de $20 \mathrm{mK}$ pour des raisons d'équilibre thermique.

Un exemple typique de point de chaleur spécifique est représenté sur la figure 7 , en fonction du temps.

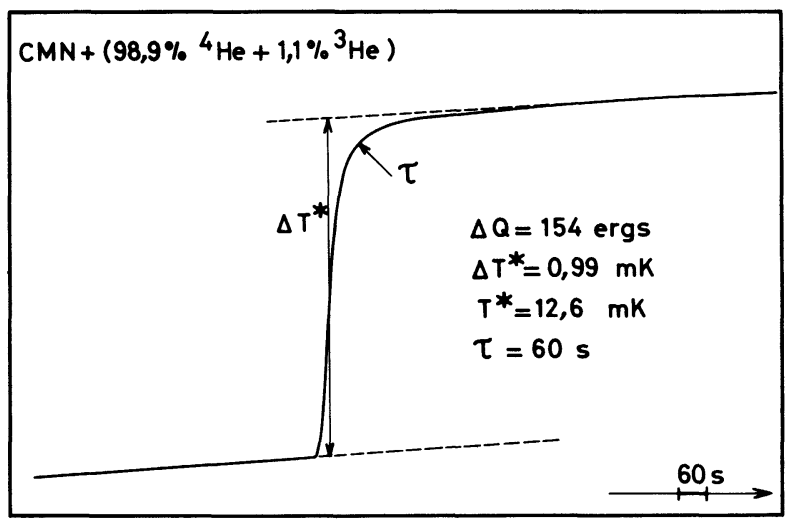

Fig. 7. - Variation en fonction du temps de la susceptibilité du CMN lors d'une mesure de chaleur spécifique.

[CMN susceptibility variation versus time during a specific heat measurement.]

Pour tenir compte des entrées de chaleur parasite, l'enregistrement est effectué pendant plusieurs dizaines de minutes avant et après chaque période de chauffage. La constante de temps de retour à l'équilibre $\tau$ liée au transfert thermique entre le CMN et l'hélium et la variation totale de susceptibilité mesurant l'élévation de température $\Delta T^{*}$ sont directement tirées de cet enregistrement.
3. Mesures et résultats. - 3.1 Mesures DE CHALEUR SPÉCIFIQUE. - 3.1.1 Mesures avec ${ }^{3} \mathrm{He}$ liquide. - Sur la figure 8 , on a représenté la capacité calorifique de la cellule remplie avec ${ }^{3} \mathrm{He}$ sous pression nulle et sous une pression de 29,2 bars, entre $1,6 \mathrm{mK}$ et $3 \mathrm{mK}$. Dans cette gamme de température, les

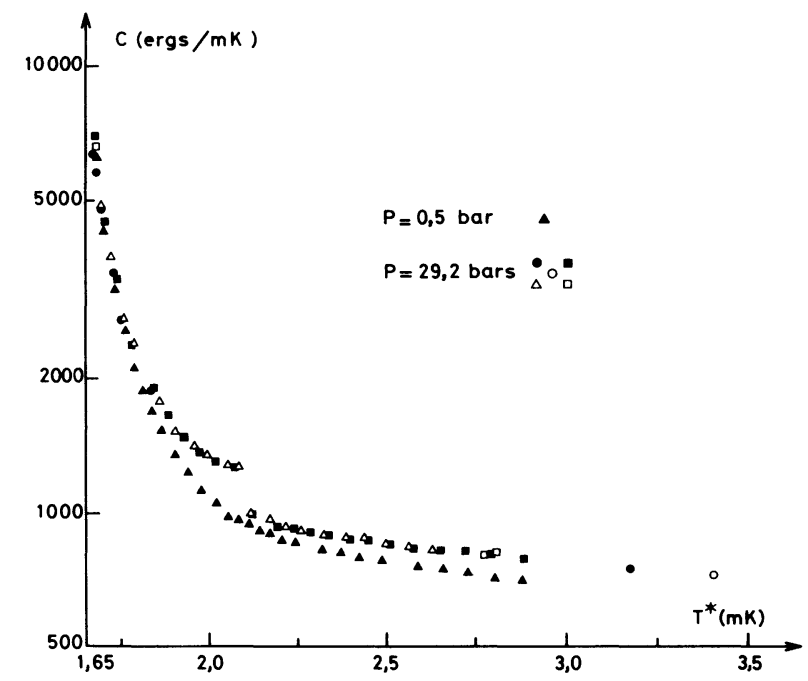

FIG. 8. - Capacité thermique de la cellule remplie de ${ }^{3} \mathrm{He}$ liquide. [Filled liquid ${ }^{3} \mathrm{He}$ cell heat capacity.]

constantes de temps de retour à l'équilibre après l'impulsion de chaleur sont de l'ordre de quelques secondes. La dérive due aux apports de chaleur parasite est constante avant et après le temps de chauffage et correspond à une entrée de chaleur d'environ $1 \mathrm{erg} / \mathrm{min}$. La dispersion des résultats est d'environ $1 \%$.

Les mesures sous pression présentent une discontinuité à $T_{\mathrm{A}}^{*}=2,1 \mathrm{mK}$ caractéristique de la transition superfluidé de ${ }^{3} \mathrm{He}$. Dans cette gamme de température, la chaleur spécifique du CMN, au moins cinq fois plus grande que celle de ${ }^{3} \mathrm{He}$, est mal connue en particulier à cause de l'incertitude sur la température absolue [7-11]. Pour séparer les capacités calorifiques du $\mathrm{CMN}$ et de ${ }^{3} \mathrm{He}$ superfluide, nous reprenons une méthode utilisée dans la référence [4].

Sous 29,2 bars, la capacité calorifique $C_{29,2}$ de la cellule est :

$$
C_{29,2}=n_{\mathrm{S}} C_{\mathrm{CMN}}+V C_{29,2}^{3}
$$

$n_{\mathrm{S}}$, nombre de moles de $\mathrm{CMN} ; V$, volume de ${ }^{3} \mathrm{He}$; $C_{\mathrm{CMN}}$ et $C_{29,2}^{3}$, chaleur spécifique par mole de CMN et par $\mathrm{cm}^{3} \mathrm{de}^{3} \mathrm{He}$.

Sous une pression de 0,5 bar, on a avec les mêmes notations :

$$
C_{0,5}=n_{\mathrm{S}} C_{\mathrm{CMN}}+V C_{0,5}^{3} .
$$

Par différence on obtient :

$$
C_{29,2}-C_{0,5}=V\left(C_{29,2}^{3}-C_{0,5}^{3}\right) .
$$


De nos mesures au-dessus de $T_{\mathrm{A}}$, on détermine le volume $V$ de ${ }^{3} \mathrm{He}$ remplissant la cellule, à partir de (5), en utilisant les tables donnant la chaleur spécifique de ${ }^{3} \mathrm{He}$ [25]. L'équation (4) nous permet de déduire le terme $n_{\mathrm{S}} C_{\mathrm{CMN}}$ présenté sur la figure 9 ;

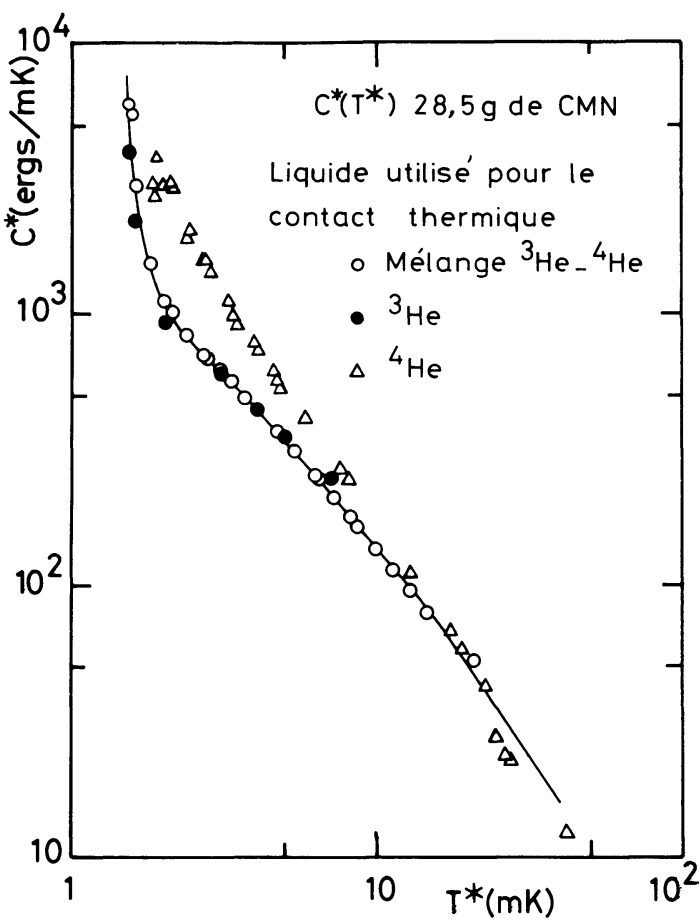

FIG. 9. - Capacité thermique de $28,5 \mathrm{~g}$ de CMN avec divers liquides comme agent thermique de contact.

[Heat capacity of $28.5 \mathrm{~g}$ of $\mathrm{CMN}$ with various liquids used for thermal contact.]

en reportant dans (3) on obtient la capacité thermique de ${ }^{3} \mathrm{He}$ présent dans la cellule dans l'état normal ou dant l'état superfluide, sous 29,2 bars. La variation en fonction de la température de cette capacité thermique est représentée sur la figure 10. Nos résultats sont en très bon accord avec ceux obtenus

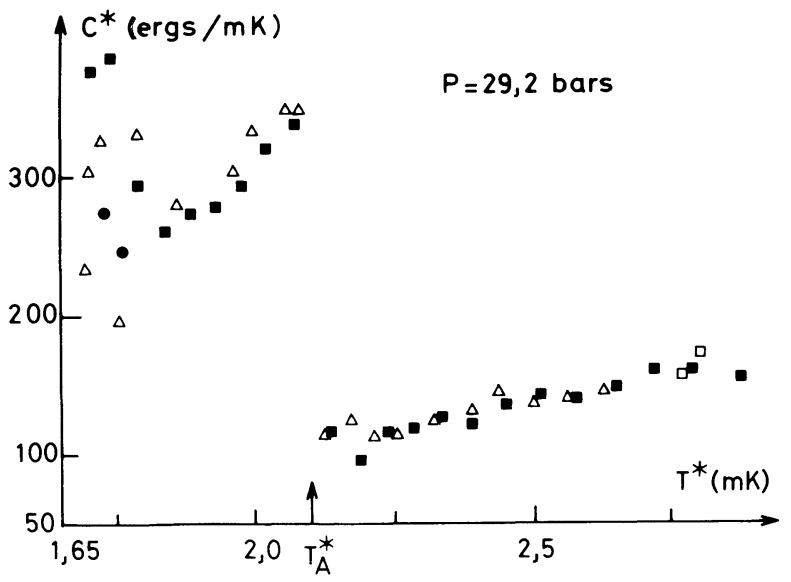

Fig. 10. - Capacité thermique de ${ }^{3} \mathrm{He}$ contenu dans la cellule sous une pression de 29,2 bars

[ ${ }^{3} \mathrm{He}$ heat capacity at a pressure of 29.2 bars.] précédemment [4]. Les échelles de température magnétique correspondent à $\pm 5 \%$.

En dessous de 1,8 $\mathrm{mK}$, il n'est plus possible de déterminer avec une bonne précision la capacité thermique de ${ }^{3} \mathrm{He}$ sous pression qui représente moins de $5 \%$ de la capacité thermique totale, ce qui explique la grande dispersion des résultats.

La mesure du saut de chaleur spécifique à la transition superfluide est faite à partir des résultats de la figure 11 où l'on a représenté la vitesse de réchauffement de la cellule $\dot{T}$ en fonction de sa température autour de $T_{\mathrm{A}}$ pour deux régimes différents :

a) En laissant la cellule se réchauffer sous l'action des pertes;

b) En ajoutant à ces pertes une puissance supplémentaire de $2,3 \mathrm{ergs} / \mathrm{min}$.

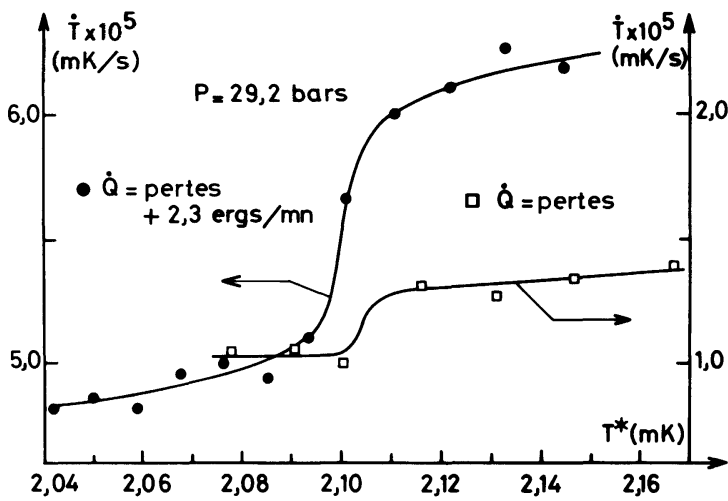

Fig. 11. - Vitesse de réchauffement de la cellule, remplie de ${ }^{3} \mathrm{He}$ à la transition superfluide $T_{\mathrm{A}}$ sous 29,2 bars en fonction de la température pour deux régimes de pertes différents.

[Filled ${ }^{3} \mathrm{He}$ cell heating rate at $T_{\mathrm{A}}$ versus temperature for two different regimes of heat leak at a pressure of 29.2 bars.]

Cette figure met bien en évidence la transition superfluide de ${ }^{3} \mathrm{He}$ qui se caractérise par une discontinuité de $\dot{T}$. La demi-largeụr de transition est d'environ $0,3 \%$. Soient $C_{>}, \dot{T}_{>}$la capacité thermique de la cellule et sa vitesse de réchauffement juste en dessus de $T_{\mathrm{A}}, \dot{T}_{<}$sa vitesse de réchauffement juste au-dessous de $T_{\mathrm{A}}, \Delta C_{3}$ l'excès de capacité thermique de ${ }^{3} \mathrm{He}$ dû à sa transition superfluide. Les pertes $\dot{Q}$ sur la cellule sont alors données par :

d'où :

$$
\dot{Q}=\left(C_{>}+\Delta C_{3}\right) \dot{T}_{<}=C_{>} \dot{T}_{>}
$$

$$
\Delta C_{3}=C_{>}\left(\frac{\dot{T}_{>}}{\dot{T}_{<}}-1\right) \text {. }
$$

On obtient alors :

- dans le régime $a$ ) : $\Delta C_{3}=235 \mathrm{ergs} / \mathrm{mK}$, $C_{3<} / C_{3>}=3$

— dans le régime b) : $\Delta C_{3}=225 \mathrm{ergs} / \mathrm{mK}$, $C_{3<} / C_{3>}=3$.

$C_{3}>$ et $C_{3<}$ étant les capacités thermiques de ${ }^{3} \mathrm{He}$ dans l'état normal et dans l'état superfluide à $T_{\mathrm{A}}$, l'incertitude sur le rapport étant d'environ $\pm 5 \%$. 
Ces résultats sont en bon accord avec ceux obtenus précédemment [4]. Ils montrent également que le confinement de ${ }^{3} \mathrm{He}$ dans des pores de taille comprise entre 1 et $5 \mu$ (observée au microscope électronique à balayage) ne modifie pas de façon sensible les résultats des mesures de chaleur spécifique effectuées dans ${ }^{3} \mathrm{He}$ massif [2]. Le rapport $C_{3<} / C_{3}>$ mesuré est supérieur à la valeur 2,59 déduite des mesures de RMN [26] dans le cadre d'une théorie de fluctuation de spin [27].

3.1.2 Mesures avec ${ }^{4} \mathrm{He}$ liquide. - Lors des mesures avec ${ }^{3} \mathrm{He}$ liquide, le terme $n_{\mathrm{S}} C_{\mathrm{CMN}}$ est mesurable jusqu'à environ $10 \mathrm{mK}$. Au-dessus de cette température, la capacité thermique de ${ }^{3} \mathrm{He}$ est trop grande par rapport à celle du $\mathrm{CMN}$. En remplaçant ${ }^{3} \mathrm{He}$ par ${ }^{4} \mathrm{He}$ liquide, on attend une contribution négligeable du liquide à la capacité thermique totale de la cellule pour $T<0,1 \mathrm{~K}$ et donc une mesure directe de la capacité thermique du CMN.

Les résultats présentés sur la figure 9 montrent un désaccord important avec les mesures effectuées avec ${ }^{3} \mathrm{He}$ en-dessous de $8 \mathrm{mK}$, allant jusqu'à un facteur 3 à $2 \mathrm{mK}$. Ces résultats ont été obtenus lors de deux séries de mesures. Dans la première, la cellule se réchauffait sous l'effet de pertes de $45 \mathrm{ergs} / \mathrm{min}$. dues à une mauvaise thermalisation de ${ }^{4} \mathrm{He}$. Un capillaire de $\mathrm{Cu}-\mathrm{Ni}\left(\varnothing_{\text {int }}=0,2 \mathrm{~mm}, L=25 \mathrm{~mm}\right)$ entre la boîte à $1 \mathrm{~K}$ et l'évaporateur constituait un court-circuit thermique [28] et les thermalisations sur l'évaporateur et la plaque froide étaient insuffisantes. Dans la seconde série de mesures, on a pu ramener les pertes sur la cellule à environ $2 \mathrm{ergs} / \mathrm{min}$. en mettant un capillaire de $\mathrm{Cu}-\mathrm{Ni}\left(\varnothing_{\text {int }}=0,1 \mathrm{~mm}\right.$, $L=27 \mathrm{~cm}$ ) entre la boîte à $1 \mathrm{~K}$ et l'évaporateur et en accroissant la surface d'échange pour les thermalisations au niveau de l'évaporateur et de la plaque froide. Les points, bien représentés par une loi en $T^{*-1,7}$ sur environ trois décades, sont reproductibles, ce qui semble éliminer l'hypothèse d'un mauvais équilibre thermique dû aux pertes aux très basses températures.

Lors d'une mesure, une quantité de chaleur comprise entre 3 et 96 ergs était dissipée pendant un temps $t$ tel que : $0,01 \mathrm{~s}<t<100 \mathrm{~s}$. La puissance de chauffage était comprise entre $0,25 \mathrm{erg} / \mathrm{s}$ et $1200 \mathrm{ergs} / \mathrm{s}$ et le rapport de l'échauffement $\Delta T$ à la température moyenne lors d'une mesure entre $0,2 \%$ et $7 \%$. La bonne reproductibilité des résultats malgré ces conditions extrêmes montre qu'il ne faut pas chercher dans ces conditions de chauffage l'origine du désaccord.

L'étalonnage du CMN a varié d'environ $10 \%$ entre les mesures avec ${ }^{3} \mathrm{He}$ et ${ }^{4} \mathrm{He}$. L'incertitude sur la mesure de la température et de l'échauffement lors d'une mesure de capacité thermique ne suffit pas à expliquer un rapport 2 à 3 dans les résultats vers $2 \mathrm{mK}$. Des mesures effectuées, après celles avec ${ }^{4} \mathrm{He}$, en utilisant un mélange ${ }^{3} \mathrm{He}-{ }^{4} \mathrm{He}$ sont en bon accord au-dessus de $10 \mathrm{mK}$ avec les mesures avec
${ }^{4} \mathrm{He}$. Dans ce cas, l'étalonnage était retrouvé égal à celui obtenu initialement avec ${ }^{3} \mathrm{He}$.

Si l'on prend pour résistance de Kapitza entre le $\mathrm{CMN}$ et ${ }^{4} \mathrm{He}$ une valeur comparable à celle du cuivre avec ${ }^{4} \mathrm{He}: R_{\mathrm{K}}=200 T^{-3} \mathrm{~cm}^{2} . \mathrm{K} / \mathrm{W}$, avec des pertes de $2 \mathrm{ergs} / \mathrm{min}$. et une surface de contact $\mathrm{CMN}-{ }^{4} \mathrm{He}$ d'environ $5 \times 10^{4} \mathrm{~cm}^{2}$, mesurée par la méthode BET, on calcule une température minimale de ${ }^{4} \mathrm{He}$ voisine de $3 \mathrm{mK}$. Avec des pertes de $45 \mathrm{ergs} / \mathrm{min}$., la température minimale de ${ }^{4} \mathrm{He}$ calculée serait voisine de $6,5 \mathrm{mK}$. Les résultats dans les deux cas sont identiques, ce qui confirme l'hypothèse d'une contribution négligeable de ${ }^{4} \mathrm{He}$ à la capacité calorifique totale de la cellule.

De même une surchauffe locale du CMN ne semble pas pouvoir expliquer le désaccord, les ordres de grandeur mis en cause étant peu réalistes (voir Annexe II).

Une fuite thermique parasite pourrait expliquer nos résultats. En effet, la quantité de chaleur $\Delta Q$ causant l'échauffement $\Delta T$ serait alors inférieure à la quantité de chaleur $\Delta Q_{\mathrm{m}}$ mesurée et la capacité calorifique déduite supérieure à la capacité calorifique réelle. La différence $\Delta Q_{\mathrm{m}}-\Delta Q$ pourrait par exemple être transférée vers la boîte à mélange via l'interrupteur thermique ou vers la plaque froide à $30 \mathrm{mK}$ via le capillaire de remplissage de la cellule plein de ${ }^{4} \mathrm{He}$ liquide. En fait, la résistance de carbone placée sur l'interrupteur thermique côté cellule s'échauffe jusqu'à 20 ou $30 \mathrm{mK}$ environ lors de l'impulsion de chaleur puis relaxe rapidement à la température d'équilibre ; ceci traduit la surchauffe de ${ }^{4} \mathrm{He}$ due à sa faible capacité thermique puis le transfert de chaleur de ${ }^{4} \mathrm{He}$ au CMN. Dans ces conditions, la quantité de chaleur pouvant être perdue via l'interrupteur thermique est de quelques dixièmes d'erg et ne peut expliquer le désaccord observé. De même les résistances placées sur la plaque froide ne montrent aucun réchauffement lié à l'impulsion de chaleur. Aucune perte d'énergie par le capillaire de remplissage ne semble donc intervenir. Tout ceci ne fait que confirmer d'ailleurs le caractère adiabatique de nos mesures.

3.1.3 Mesures avec un mélange ${ }^{3} \mathrm{He}-{ }^{4} \mathrm{He}$ - Devant ce désaccord, nous avons entrepris deux séries de mesures avec un mélange $98,9 \%{ }^{4} \mathrm{He}-1,1 \%{ }^{3} \mathrm{He}$. La température de Fermi du mélange étant beaucoup plus faible que celle de ${ }^{3} \mathrm{He}$ pur, sa capacité thermique est également plus faible. Ceci nous permet de mesurer la capacité thermique du CMN en-dessous d'environ $20 \mathrm{mK}$. Au-delà, la capacité thermique du mélange est trop grande par rapport à celle du CMN pour que l'on puisse faire des mesures précises.

Les résultats sont également présentés sur la figure 9 et sont en très bon accord avec ceux obtenus avec ${ }^{3} \mathrm{He}$. Ils sont déduits des mesures de capacité thermique en utilisant les valeurs extrapolées de la référence [29] pour la chaleur spécifique du mélange. 
3.1.4 Discussion des résultats obtenus sur la capacité thermique du CMN. - Nos mesures de capacité thermique du $\mathrm{CMN}$ en présence de ${ }^{3} \mathrm{He}$ ou d'un mélange ${ }^{3} \mathrm{He}-{ }^{4} \mathrm{He}$ montrent une variation en $T^{*-1,7}$ au-dessus de $10 \mathrm{mK}$ environ, plus faible jusqu'à $5 \mathrm{mK}$, en bon accord avec d'autres résultats $[7,9,10$, 11] obtenus par la même méthode de mesure directe de la capacité thermique. Aux températures inférieures à $7 \mathrm{mK}$ les comparaisons deviennent plus difficiles à faire, les échelles de température pouvant subir des distorsions importantes lorsqu'on se rapproche de la température d'ordre du CMN. Les résultats obtenus par différentiation de courbes entropie-température [8] donnent également une capacité thermique en accord raisonnable avec nos résultats au-dessus de $10 \mathrm{mK}$, limite inférieure de leurs valeurs.

Il faut noter que ces mesures sont faites sur des échantillons très divers : massifs $[8,10,11]$, grains de diamètre inférieur à $420 \mu[7]$ ou, dans notre cas, grains de diamètre moyen $30-40 \mu$.

L'influence des impuretés semble aussi peu importante : le CMN utilisé dans nos mesures contient 500 ppm de Pr, 65 ppm d'Er, moins de 5 ppm d'Yb. D'autres échantillons $[8,11]$ ont un taux d'impureté très différent et donnent les mêmes résultats lors des mesures de capacité thermique pour des températures inférieures à $25 \mathrm{mK}$. Ceci confirme que pour ces températures un modèle de dipôles en interaction rend bien compte des résultats, l'influence des impuretés en particulier $\operatorname{Pr}$ n'intervenant qu'à plus haute température [30]. L'ensemble de ces résultats montre qu'une erreur grossière dans la façon dont sont conduites les mesures et analysés les résultats ne semble pas être à l'origine du désaccord observé lors des expériences avec ${ }^{4} \mathrm{He}$ liquide. Des mesures plus anciennes [7] ont donné des résultats similaires qui étaient attribués à un défaut d'équilibre de température dans la cellule. Ceci ne semble pas pouvoir être retenu dans nos expériences.

On est ainsi conduit à envisager une possible origine physique à ce désaccord. Diverses approches ont été envisagées dans ce sens.

Une hypothèse, envisageant l'existence à très basse température d'interactions (à déterminer) entre le $\mathrm{CMN}$ et un liquide de Fermi $\left({ }^{3} \mathrm{He}\right.$ ou un mélange ${ }^{3} \mathrm{He}-$ ${ }^{4} \mathrm{He}$ ), semble devoir être rejetée. En effet, certaines mesures [10], qui ont été effectuées sans que l'échantillon ait été mis en présence de ${ }^{3} \mathrm{He}$, sont en bon accord avec nos résultats avec ${ }^{3} \mathrm{He}$.

On peut évaluer le nombre minimum d'atomes concernés par l'anomalie de chaleur spécifique d'environ 2000 ergs $/ \mathrm{mK}$ à $2 \mathrm{mK}$, en estimant à $R$ l'ordre de grandeur maximum de la capacité thermique pour une mole. On trouve alors

$$
2,4 \times 10^{-2} \text { ion-gramme }
$$

de CMN ou mole de ${ }^{4} \mathrm{He}$ alors que la cellule contient $3,8 \times 10^{-2}$ ion-gramme de CMN et 0,14 mole de
${ }^{4} \mathrm{He}$. Ces valeurs montrent qu'un effet de surface doit être écarté et que seul un effet dans toute la masse de l'échantillon peut éventuellement prendre en compte l'anomalie.

Une autre hypothèse suppose que la vraie chaleur spécifique du CMN est celle mesurée lors des expériences avec ${ }^{3} \mathrm{He}$ ou le mélange. Pendant une impulsion de chaleur, ${ }^{4} \mathrm{He}$ se trouverait porté localement à haute température ( $1 \mathrm{~K}$ voire beaucoup plus) et les excitations ainsi créées se trouvent bloquées dans un état métastable lors du retour à l'équilibre thermique. A $2 \mathrm{mK}$, l'excès de capacite thermique dans nos mesures avec ${ }^{4} \mathrm{He}$ est d'environ $2000 \mathrm{ergs} / \mathrm{mK}$ correspondant par exemple à celle de rotons à une température de $1,4 \mathrm{~K}$ [19]. Néanmoins une telle interprétation semble difficile à soutenir. Comment des excitations peuvent-elles subsister dans un état métastable à basse température et avoir une chaleur spécifique variant avec la température du CMN, devenant négligeable vers $10 \mathrm{mK}$ ? De plus, un tel système devrait présenter un caractère très hystérétique dépendant en particulier de la valeur de la puissance dégagée pendant l'impulsion de chaleur. Ceci n'est pas le cas comme il a déjà été souligné : lors des diverses séries de mesures, nous avons observé une très bonne reproductibilité des résultats quelles que soient les caractéristiques de l'impulsion de chaleur.

Le spectre des excitations de ${ }^{4} \mathrm{He}$ à aussi basse température est très mal connu. Il semble néanmoins bien improbable qu'il présente une anomalie suffisamment grande pour expliquer le désaccord observé dans nos mesures. L'influence de cette anomalie devrait être observable bien au-dessus de $10 \mathrm{mK}$. De plus comme on l'a déjà vu, la reproductibilité des résultats, lors des mesures avec ${ }^{4} \mathrm{He}$, malgré des pertes très différentes, indique que la contribution de ${ }^{4} \mathrm{He}$ à la chaleur spécifique totale est négligeable.

L'origine physique du désaccord semble donc très spéculative et une origine expérimentale non triviale. Heureusement dans nos expériences de transfert thermique, la capacité thermique du CMN n'intervient dans l'analyse des mesures qu'au-dessus d'environ $8 \mathrm{mK}$. Nos résultats expérimentaux sont alors en bon accord quel que soit le liquide assurant le contact thermique.

Pour éclaircir le problème en-dessous de cette température, des mesures systématiques avec des tailles de grains et des taux de compactage différents sont nécessaires. L'utilisation de CMN dilué au lanthane pourrait également fournir des résultats intéressants en diminuant les interactions magnétiques entre atomes de cérium.

3.2 MESURes DE TRANSFERT THERMiQue ENTRE CMN ET UN MÉLANGE ${ }^{3} \mathrm{He}-{ }^{4} \mathrm{He}$. - Le retour à l'équilibre lors d'une mesure de la chaleur spécifique du système $\mathrm{CMN}+\left(98,9 \%{ }^{4} \mathrm{He}+1,1 \%{ }^{3} \mathrm{He}\right)$ était quasi exponentiel. La variation thermique de la cons- 


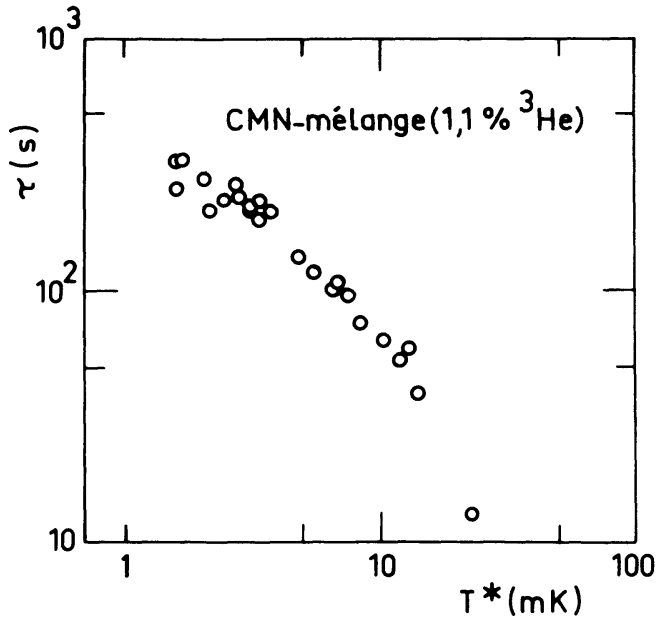

FIG. 12. - Constante de temps de retour à l'équilibre du système CMN + $\left(98,9 \%{ }^{4} \mathrm{He}+1,1 \%{ }^{3} \mathrm{He}\right)$ en fonction de la température. [Time constant of the system CMN $+\left(98.9 \%{ }^{4} \mathrm{He}+1.1 \%{ }^{3} \mathrm{He}\right)$ as a function of the temperature.]

tante de temps correspondante est présentée sur la figure 12 entre 4 et $25 \mathrm{mK}$. Dans un modèle simple où l'on considère le $\mathrm{CMN}$ et le mélange couplés par une résistance thermique $R$, on a la relation :

$$
\tau=R \bar{C} \text { avec } \bar{C}=\frac{C_{\mathrm{CMN}} C_{\mathrm{m}}}{C_{\mathrm{CMN}}+C_{\mathrm{m}}},
$$

où $C_{\mathrm{CMN}}$ et $C_{\mathrm{m}}$ représentent respectivement les capacités thermiques du CMN et du mélange. Comme on l'a déjà remarqué pour $T \lesssim 12 \mathrm{mK}$ la capacité thermique du mélange est faible par rapport à celle

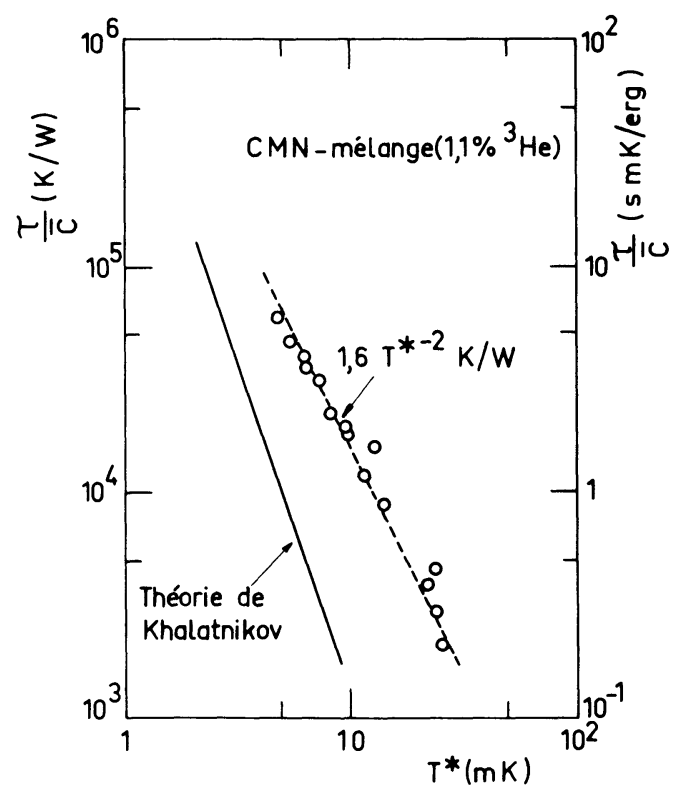

FIG. 13. $-\frac{\tau}{\bar{C}}$ en fonction de la température dans le système $\mathrm{CMN}+\left(98,9 \%{ }^{4} \mathrm{He}+1,1 \%{ }^{3} \mathrm{He}\right)$.

$\left[\frac{\tau}{\bar{C}}\right.$ as a function of the temperature in the system

$$
\left.\mathrm{CMN}+\left(98.9 \%{ }^{4} \mathrm{He}+1.1 \%{ }^{3} \mathrm{He}\right) .\right]
$$

du CMN et en dessous de $10 \mathrm{mK}$ on peut écrire : $\tau \simeq R C_{\mathrm{m}}$. Par conséquent, les problèmes rencontrés dans la mesure de la capacité thermique du CMN en dessous de $10 \mathrm{mK}$ ne rejaillissent pas dans l'analyse du transfert thermique entre le $\mathrm{CMN}$ et le mélange. Le rapport $\tau / \bar{C}$ est représenté sur la figure 13 entre 4 et $25 \mathrm{mK}$. La variation thermique suit une loi en $T^{*-2}$. Sur la même figure on a représenté la loi en $T^{-3}$ décrivant la variation théorique attendue dans un modèle de désaccord acoustique [31]. Nos résultats ne présentent pas la variation thermique attendue, mais l'ordre de grandeur en-dessous de $10 \mathrm{mK}$ est celui prévu par la théorie. Des résultats montrant une résistance de contact mesurée bien plus élevée que celle calculée théoriquement ont aussi été plusieurs fois présentés dans l'étude du transfert thermique entre une poudre et l'hélium [20, 32] mais restent encore sans explication.

En fait cette situation est inhabituelle. Dans les mesures de résistance de Kapitza au voisinage de $1 \mathrm{~K}$, les valeurs mesurées sont de façon générale plus faibles que les valeurs déduites de la théorie et n'ont pas la dépendance de $T$ attendue, qui ne commence à être suivie qu'aux plus basses températures $(T \lesssim 100 \mathrm{mK})$.

Les modifications éventuelles dans notre arrangement expérimental des paramètres utilisés dans la théorie (vitesse du son, température de Debye par exemple) ne peuvent pas rendre compte quantitativement de l'écart entre théorie et expérience : dans des grains de faibles dimensions compactés très fortement, les variations attendues de ces paramètres ne sont que de l'ordre du pour cent et n'expliquent pas le rapport d'environ huit mesuré ici.

En fait d'autres mesures utilisant des grains de CMN de taille différente sont nécessaires pour déterminer l'origine de cette situation assez exceptionnelle dans l'étude du transfert thermique à basse température.

4. Conclusion. - Nous avons mis au point un appareil permettant des mesures de chaleur spécifique et de transfert thermique jusqu'à la température d'ordre du CMN. Les mesures de chaleur spécifique à la transition superfluide de ${ }^{3} \mathrm{He}$ liquide sont en bon accord avec les mesures antérieures. Les mesures ont aussi montré un comportement anormal de la chaleur spécifique en présence de ${ }^{4} \mathrm{He}$ liquide, en dessous de $10 \mathrm{mK}$. D'autres expériences sont nécessaires pour en déterminer l'origine.

Les mesures de transfert thermique montrent que le modèle utilisé pour l'analyse des constantes de temps nous donne des résultats en accord avec ceux obtenus dans d'autres expériences au contact poudrehélium. Le désaccord avec les prévisions du processus de phonons classiques n'a pas une origine claire. Ce travail est le point de départ d'une étude du transfert thermique avec ${ }^{3} \mathrm{He}$ liquide ou solide dont les résultats seront publiés par ailleurs. 
Remerciements. - Nous remercions M. Jurek pour l'aide qu'il nous a apportée dans la construction et la mise au point de notre appareil et Mlle Amiot et M. Palleau qui ont préparé le CMN et effectué son analyse par absorption atomique. M. Ginoux de l'ENSEEG a mesuré la surface spécifique du CMN.

Annexe I. - Caractéristiques de la ligne DE POMPAGE DU MÉLANGE. - Le gaz est pompé sur l'évaporateur à travers un tube de $\varnothing 6 \mathrm{~mm}$ et de $3 \mathrm{~cm}$ de long. Le chauffage destiné à brûler le film de ${ }^{4} \mathrm{He}$ superfluide se trouve sur ce tube qui est donc maintenu à une température supérieure ou égale à $T_{\lambda}$; ce brûleur de film permet également de chauffer l'évaporateur pour accroître le débit du gaz.

Les caractéristiques des tubes de pompage (en acier inoxydable) dans le cryostat sont rassemblées dans le tableau II. $L$ est la longueur du tube en $\mathrm{cm}$;

\section{TABLEAU II}

Caractéristiques de la ligne de pompage du mélange. $L$ longueur du tube en $\mathrm{cm} ; \varnothing_{\mathrm{i}}, \varnothing_{\mathrm{e}}$ diamètres intérieur et extérieur du tube en $\mathrm{mm} ; T_{\mathrm{s}}, T_{\mathrm{i}}$ températures des extrémités supérieure et inférieure du tube en $\mathrm{K}$.

[Mixture pumping line characteristics. $L$ tube length in $\mathrm{cm} ; \varnothing_{\mathrm{i}}, \varnothing_{\mathrm{e}}$ inner and outer tube diameters in $\mathrm{mm} ; T_{\mathrm{s}}, T_{\mathrm{i}}$ upper and lower tube ends temperatures in $\mathrm{K}$.]

$\begin{array}{ccccl}L \mathrm{~cm} & \varnothing_{\mathrm{i}} \mathrm{mm} & \varnothing_{\mathrm{e}} \mathrm{mm} & T_{\mathrm{s}} \mathrm{K} & T_{\mathrm{i}} \mathrm{K} \\ \overline{15} & - & - & - & - \\ 20 & 13 & 14 & 4,2 & 0,6\left(^{1}\right) \\ 25 & 29 & 30 & 4,2-20 & 4,2 \\ 35 & 60 & 61 & 80-130 & 4,2-20 \\ \text { Coude } & 90 & 91,5 & 300 & 80-130 \\ 200 & 125 & & & \end{array}$

( $\left.{ }^{1}\right)$ Egalement thermalisé sur la boîte à $1,2 \mathrm{~K}$.

$\varnothing_{\mathrm{i}}$ et $\varnothing_{\mathrm{e}}$, ses diamètres intérieur et extérieur en $\mathrm{mm}$; $T_{\mathrm{s}}$ et $T_{\mathrm{i}}$, les températures de ses extrémités supérieure et inférieure en $\mathbf{K}$. La ligne de pompage hors du cryostat comporte des soufflets inox, pour limiter l'effet des vibrations, montés de façon compensée. Cet ensemble est relié à la pompe à diffusion Bendix KS 200 via un piège à azote et un baffle à eau destinés à minimiser l'effet d'une éventuelle rétrodiffusion de l'huile utilisée (Convoil 10). Le piège et le baffle ont un effet négligeable sur les caractéristiques de la pompe.

Un calcul de conductance classique montre que chacune des sections de la ligne de pompage sauf le tube $\varnothing 6 \mathrm{~mm}$ de l'évaporateur donne lieu à la même perte de charge.

Il montre en particulier qu'il est indispensable de thermaliser le tube $\varnothing 13-14$ sur la boîte à $1,2 \mathrm{~K}$ et que le point critique de la ligne est le tube de pompage de l'évaporateur. Pour éviter ce problème, les évaporateurs utilisés maintenant au laboratoire disposent de deux chauffages : l'un sur le tube de pompage brûle le film, le tube restant à environ $T_{\lambda}$; le second est placé dans le liquide dilué et le chauffe à la température d'ébullition convenable pour assurer le débit souhaité. Cet arrangement évite la surchauffe du tube $\varnothing 6 \mathrm{~mm}$ qui dans notre cas est à l'origine d'une perte de charge limitant les performances du système de pompage.

Annexe II. - Surchauffe locale du CMN lors DES EXPÉRIENCES AVEC ${ }^{4} \mathrm{He}$. - La taille des pores dans nos expériences est inférieure à $5 \mu$. La conductivité thermique de ${ }^{3} \mathrm{He}$ ou du mélange au voisinage du mK est très bonne [2]; aucune mesure sur ${ }^{4} \mathrm{He}$ liquide n'a été faite dans cette gamme de température. Le fil de chauffage dans la cellule est localisé et une éventuelle surchauffe locale du CMN due à une mauvaise diffusion de la chaleur dans les canaux remplis de ${ }^{4} \mathrm{He}$ peut donner lieu à un écart sensible lors de la mesure de l'échauffement $\Delta T$ correspondant à une énergie $\Delta Q$ dissipée dans la cellule, faussant les mesures.

Soient $C$ la capacité thermique totale de la cellule à la température $T$, remplie de ${ }^{4} \mathrm{He}$, et $\Delta T$ l'échauffement de la cellule en l'absence de surchauffe : $\Delta Q=C \Delta T$. La mutuelle va nous donner une valeur initiale : $M_{\mathrm{i}}=\frac{a}{T}+b$ et une valeur finale après échauffement : $M_{\mathrm{f}}=\frac{a}{T+\Delta T}+b$

$$
\Delta M=M_{\mathrm{f}}-M_{\mathrm{i}}=-\frac{a \Delta T}{T^{2}(1+\Delta T / T)} .
$$

En cas de surchauffe locale on ne chauffe que $x \%$ du CMN et $\Delta Q=x C \frac{\Delta T}{x}$. La valeur initiale de la mutuelle sera : $M_{\mathrm{i}}=\frac{a}{T}+b$, sa valeur finale :

et

$$
M_{\mathrm{f}}=\frac{(1-x) a}{T}+\frac{x a}{T+\frac{\Delta T}{x}}+b
$$

$$
\Delta M=-\frac{a \Delta T}{T^{2}\left(1+\frac{\Delta T}{x T}\right)} .
$$

Les deux valeurs obtenues (6) et (7) sont identiques dans la mesure où : $\Delta T / x T \ll 1(8)$. Dans nos expériences, en général : $\Delta T / T \sim 1 \%$ et alors (8) n'est plus vérifié si $x<10 \%$. Néanmoins certains pơints sont tels que $\Delta T / T \sim 10 \%$ et la condition (8) est alors beaucoup plus stricte. Dans les deux cas, les mesures donnent des résultats identiques ce qui indique que le processus envisagé n'explique pas le désaccord observé à basse température entre les mesures avec ${ }^{3} \mathrm{He}$ et celles avec ${ }^{4} \mathrm{He}$. 


\section{Bibliographie}

[1] Peshkov, V. P., Zh. Eksperim. i Teor. Fiz. 48 (1965) 997 ; (English transl. : Sov. Physics JETP 21 (1965) 663).

[2] Wheatley, J. C., "Quantum Fluids », D. F. Brewer, Editor, (North-Holland Publishing Co., Amsterdam) 1966, 183.

[3] Wheatley, J. C., Vilches, O. E., Abel, W. R., Physics 4 (1968) 1.

[4] WebB, R. A., Greytak, T. J., Johnson, R. T., Wheatley, J. C., Phys. Rev. Lett. 30 (1973) 210.

[5] Ahonen, A. I., Haikala, M. T., Krusius, M., LounasMaA, O. V., Phys. Rev. Lett. 33 (1974) 628.

[6] Osheroff, D. D., Gully, W. J., Richardson, R. C., Lee, D. M., Phys. Rev. Lett. 29 (1972) 920.

[7] Wheatley, J. C., Ann. Acad. Sci. Fenn. A 210 (1966) 15.

[8] Hudson, R. P., Kaeser, R. S., Physics 3 (1967) 95.

[9] Abraham, B. M., Eckstein, Y., Phys. Rev. Lett. 20 (1968) 649.

[10] Mess, K. W., Lubbers, J., Niesen, L., Huiskamp, W. J., Physica 41 (1969) 260.

[11] Fisher, R. A., Hornung, E. W., Brodale, G. E., Giauque, W. F., J. Chem. Phys. 58 (1973) 5584.

[12] Laumond, Y., Thèse Docteur-Ingénieur, Grenoble (1972).

[13] Anderson, A. C., Rev. Sci. Instrum. 41 (1970) 1446.

[14] Del Castillo, L., Frossati, G., Lacaze, A., Thoulouze, D., Proc. 13th Int. Conf. on Low Temp. Phys. (Plenum Press, New York) 1973.

[15] Fourni par Du Pont de Nemours, 9, rue de Vienne, 75008 Paris.

[16] Produit par Schjeldahl, Eastern Rd, Bracknell, Berksh, Angleterre.

[17] Produit par Sames, 21, rue Jean-Macé, 38000 Grenoble.

[18] Hebral, B., Matho, K., Mignot, J. M., Tournier, R., J. Physique Lett. 38 (1977) L-347.
[19] WILKs, J., The properties of liquid and solid Helium (Clarendon Press, Oxford) 1967.

[20] Frossati, G., Godfrin, H., Hebral, B., Schumacher, G., Thoulouze, D., Proceedings of the Symposium on ULT Physics, Hakone, Japan (1977) 205.

[21] Radebaugh, R., Siegwarth, J. D., Proceedings of the Symposium on ULT Physics, Hakone, Japan (1977) 303.

[22] Anderson, A. C., Connolly, J. I., Wheatley, J. C., Phys. Rev. 135 (1964) A910.

[23] Kodama, T., Moril, Y., Fuji, Y., Kubota, M., Shigi, T., Proc. 14th Int. Conf. on Low Temp. Phys. (North-Holland Publishing Co., Amsterdam) 1975.

[24] Fabriqué par Barras Provence, 35, quai du Drac, 38600 Fontaine, commercialisé par Oxford Instruments, sous licence ANVAR.

[25] Wheatley, J. C., The helium liquids, Proceedings of the 15th Scottish Universities Summer School in Physics, Edited by J. G. M. Armitage and I. E. Farquhar (Academic Press) 1975, 241.

[26] Osheroff, D. D., Anderson, P. W., Phys. Rev. Lett. 33 (1974) 686.

[27] Brinkman, W. F., Serene, J., Anderson, P. W., Phys. Rev. A 10 (1974) 2386.

[28] Bertman, B., Kitchens, T. A., Cryogenics 8 (1968) 36.

[29] Whentley, J. C., Progress in Low Temperature Physics, C. J. Gorter, Editor (North-Holland Publishing Co., Amsterdam) 1970, 77.

[30] Folinsbee, J. T., Harrison, J. P., McColl, D. B., J. Low Temp. Phys. 27 (1977) 25.

[31] Khalatnikov, I. M., Zh. Eksp. Teor. Fiz. 22 (1952) 687.

[32] Bishop, J. H., Cutter, D. W., Mota, A. C., Wheatley, J. C., J. Low Temp. Phys. 10 (1973) 379. 